\title{
Progranulin mutations and amyotrophic lateral sclerosis or amyotrophic lateral sclerosis-frontotemporal dementia phenotypes
}

\author{
J C Schymick, Y Yang, P M Andersen, J P Vonsattel, M Greenway, P Momeni, J Elder, A Chiò, \\ G Restagno, W Robberecht, C Dahlberg, O Mukherjee, A Goate, N Graff-Radford, R J Caselli, \\ M Hutton, J Gass, A Cannon, R Rademakers, A B Singleton, O Hardiman, J Rothstein, J Hardy, \\ B J Traynor
}

Objective: Mutations in the progranulin (PGRN) gene were recently described as the cause of ubiquitin positive frontotemporal dementia (FTD). Clinical and pathological overlap between amyotrophic lateral sclerosis (ALS) and FTD prompted us to screen PGRN in patients with ALS and ALS-FTD.

Methods: The PGRN gene was sequenced in 272 cases of sporadic ALS, 40 cases of familial ALS and in 49 patients with ALS-FTD.

Results: Missense changes were identified in an ALS-FTD patient (p.S120Y) and in a single case of limb onset sporadic ALS (p.T182M), although the pathogenicity of these variants remains unclear.

Conclusion: PGRN mutations are not a common cause of ALS phenotypes.

\footnotetext{
A
} myotrophic lateral sclerosis (ALS) is a neurodegenerative disorder predominantly involving motor neurons leading to paralysis and death within 3-5 years from symptom onset. The pathogenic mechanism leading to motor neuron degeneration is unknown in the majority of cases. Frontotemporal dementia (FTD) is a degenerative disorder of the frontal and anterior temporal lobes. ${ }^{1}$ Clinical, pathological and genetic data suggest that ALS and FTD form a spectrum of disease. ${ }^{2}$ Approximately $5 \%$ of ALS patients have FTD (ALSFTD $)^{3}$ and approximately half of patients with "classical" ALS have subtle frontal and temporal lobe cognitive impairment. ${ }^{4}$ Many FTD cases similarly develop symptoms of motor neuron involvement during the course of their illness ${ }^{5}$ and up to one third of FTD patients without overt motor symptoms have loss of anterior horn cells with characteristic ubiquitin inclusions in surviving motor neurons on autopsy. ${ }^{6}$

Recently, mutations in the progranulin (PGRN) gene have been described as the cause of ubiquitin positive FTD. ${ }^{78}$ The overlap between ALS and FTD prompted us to screen 272 cases of sporadic ALS, 40 familial ALS cases and 49 patients diagnosed with ALS-FTD for mutations in this gene.

\section{METHODS \\ Subjects}

Genetic studies were approved by local research ethics committees (NIA IRB protocol \#2003-081). Diagnosis of ALS was based on the El Escorial diagnostic criteria. ${ }^{9}$ Samples used are outlined in table 1 . The samples from the Coriell NINDS DNA repository consisted of 45 Caucasian women and 89 Caucasian men, with an average age of symptom onset of 54.3 years (range $26-81$ ). Of these, $34(25.4 \%)$ had bulbar onset disease, $98(73.1 \%)$ had limb onset weakness and the remaining $2(1.5 \%)$ cases presented with respiratory symptoms. Three sporadic ALS patients were reported to have cognitive changes, but did not have a formal diagnosis of FTD or dementia.

Of the 48 Irish cases, 20 were women and 28 were men. Average age of symptom onset was 58.9 years (range 29-79). Eight $(16.7 \%)$ patients initially manifested symptoms in bulbar musculature, $38(79.2 \%)$ cases reported limb onset symptoms and the site of onset was unspecified in the remaining $2(4.1 \%)$ cases.

Samples obtained from the brain banks at Johns Hopkins University, Columbia University and the University of Miami, consisted of 26 women and 40 men (missing data $=15$ ), with an average onset of 65.5 years of age (range 27-88, missing data $=16)$.

Of the 96 Swedish patients, 49 were males and 47 females. Thirty-one had bulbar onset motor neuron disease and 63 had spinal onset motor neuron disease. Two presented with cognitive symptoms. The mean age of onset of first symptom was 56.2 years (range 16-85). Diagnosis of FTD was based on the consensus Manchester-Lund clinical diagnostic criteria, ${ }^{10}$ supported by ancillary investigations. Of the 36 Swedish ALSFTD cases, 30 patients had ALS with FTD, four manifested ALS with language disorders characteristic of the FTD spectrum (ie, semantic dementia or progressive non-fluent aphasia) and two cases had ante mortem diagnoses of "pure" FTD with loss of anterior horn cells evident on autopsy (ie, FTD-motor neuron disease). Both of these patients had siblings or parents with ALS. North American control DNA samples were obtained from the Coriell Institute for Medical Research ( $\mathrm{n}=159$, NDPT002, NDPT006, NDPT009) together with 250 additional North American controls ascertained at the Mayo Clinic in Jacksonville, Florida and Scottsdale, Arizona.

\section{PCR amplification and sequencing of PGRN}

DNA was extracted from blood using the Wizard purification kit (Promega Corp., Wisconsin, USA) or from brain tissue using the DNeasy kit (Qiagen, Inc., California, USA). The 12 coding exons of $P G R N$ and at least $30 \mathrm{bp}$ of flanking intronic sequence were PCR amplified using primer pairs listed in appendix 1 (see http://www.jnnp.com/supplemental) and Roche FastStart PCR Master Mix polymerase (Roche Diagnostics Corp., Indiana, USA). Each product was sequenced using forward and reverse primers with Applied Biosystems BigDye terminator v3.1 sequencing chemistry.

Abbreviations: ALS, amyotrophic lateral sclerosis; FTD, frontotemporal dementia 
Table 1 Samples in which the PGRN gene was sequenced

\begin{tabular}{lcccc}
\hline & Total & SALS & FALS & ALS-FTD \\
\hline North American ALS samples* & 134 & 132 & 2 & 0 \\
Johns Hopkins Brain Bank ALS samples & 51 & 46 & 5 & 0 \\
Swedish ALS samples & 96 & 30 & 30 & 36 \\
New York Brain Bank at Columbia University ALS samples & 23 & 16 & 0 & 7 \\
University of Miami/NPF Brain Bank ALS samples & 7 & 7 & 0 & 0 \\
Irish ALS-FTD and ALS samples & 48 & 41 & 4 & 4 \\
Italian ALS-FTD sample & 1 & 0 & 0 & 1 \\
Belgium ALS-FTD sample & 1 & 0 & 0 & 1 \\
Total & 361 & 272 & 40 & 49 \\
\hline ALS, amyotrophic lateral sclerosis; FALS, familial amyotrophic lateral sclerosis; FTD, frontotemporal dementia; NPF, \\
National Parkinson Foundation; SALS, sporadic amyotrophic lateral sclerosis. \\
*http://ccr.coriell.org/ninds/catalog/panel/(sample IDs available on request). \\
\hline
\end{tabular}

\section{RESULTS}

Sequence analysis of the PGRN gene identified novel heterozygous genetic variants in a single patient diagnosed with ALSFTD $(2.0 \%, 1$ of 49$)$ and in a single case of limb onset sporadic ALS (0.4\%, l of 272 sporadic cases). Mutations were not present in the 40 familial ALS cases (see appendix 2 at http:// www.jnnp.com/supplemental).

A single nucleotide change, c.578C $>$ A, was identified in an ALS-FTD patient (New York Brain Bank sample T-51) predicting a serine to tyrosine substitution (p.S120Y, RefSeq NM_002087.2). A different nucleotide change, c.764C $>\mathrm{T}$, was identified in a patient diagnosed with sporadic ALS (Coriell ALS repository ND10418) predicting a threonine to methionine substitution at residue 182 (p.T182M). S120Y and T182M were not identified in 818 North American control chromosomes, in public databases of single nucleotide polymorphisms or in any of the other 361 ALS/ALSFTD samples screened as part of this study, or in 523 previously published FTD samples. ${ }^{711}$ A c.1395 A $>$ C base pair change was present in a single Swedish ALS case. Although this variant was also not found in 818 control chromosomes, it is unlikely to be pathogenic as it does not alter the amino acid at this codon (ie, synonymous mutation, p.Pro392Pro). Autopsy of sample T-51 revealed prominent frontal atrophy with marked neuronal loss and reactive gliosis involving the motor cortex, hypoglossal nucleus and anterior horn cells (see supplementary fig l at http:// www.jnnp.com/supplemental). Rare motor neurons showed Bunina bodies. Myelin loss was marked within the lateral and ventral corticospinal tracts.

\section{DISCUSSION}

We found variants in the PGRN gene in a single case of ALSFTD and in a single case of limb onset sporadic ALS, but mutations were not found in the other 271 sporadic ALS, 40 familial ALS or in the 48 additional ALS-FTD samples screened as part of this study. These findings suggest that PGRN mutations are not a common cause of motor neuron degeneration, although the data do not exclude the possibility that PGRN mutations may be relevant in other populations/ethnicities. Furthermore, the possibility of genomic insertion/deletion mutations in ALS patients has not been excluded by this study.

Our findings agree with a previous report that failed to find PGRN mutations in 48 ALS patients (29 sporadic cases and 19 familial cases). ${ }^{11}$ To date, only one other individual with a PGRN mutation (family UBC-17, individual 60) fulfilled the El Escorial criteria for ALS and it appears that mutations in this gene are most commonly associated with a behavioural FTD syndrome with possible parkinsonism features in the later stages of illness. ${ }^{7}{ }^{11}$ The paucity of $P G R N$ mutations in ALS patients is surprising given the clinicopathological evidence indicating that these two ubiquitionopathy syndromes overlap. However, available data indicate that the pathogenic mechanisms of ALS and FTD do not overlap completely and each individual FTD or ALS causing gene will display its own phenotype pattern.

The nature of the S120Y and T182M variants suggests that they are not pathogenic. All previously described PGRN mutations are truncating mutations that are thought to lead to nonsense mediated decay of the mutant mRNA and consequently haploinsufficiency due to loss of functional PGRN protein. ${ }^{711}$ The only exception is a c.26C $>$ A point mutation predicting a p.A9D mutation in the signal peptide of the PGRN protein that may lead to incorrect localisation of the PGRN peptide within the neuron. ${ }^{11}{ }^{12}$ Neither the S120Y nor the T182M mutations are within the signalling domain or located within any of the tandem repeats of 12 cysteines, and these residues are not highly conserved across species. Furthermore, it was not possible to demonstrate segregation of the S120Y mutation with disease, as additional DNA samples were not available from this family. However, S120Y and T182M were not found in 818 control chromosomes, indicating that they are not common polymorphisms.

In summary, we identified missense nucleotide variants in the PGRN gene in a single case of FTD associated with motor neuron dysfunction (S120Y) and in a single case of limb onset sporadic ALS (T182M), although the pathogenicity of these variants remains unclear. We conclude that PGRN mutations are not a common cause of ALS phenotypes.

\section{ACKNOWLEDGEMENTS}

We gratefully acknowledge the assistance of the New York Brain Bank-The Taub Institute, Columbia University (Federal grant No P50 AG08702) and the University of Miami/National Parkinson Foundation Brain Endowment Bank (funded by the National Parkinson Foundation, Inc., Miami, Florida, USA and other private donations).

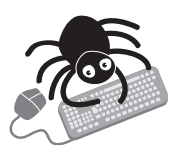

Appendices 1 and 2, and supplementary data can be viewed on the $J$ Neurol Neurosurg Psychiatry website at http://www.jnnp.com/supplemental.

\footnotetext{
Authors' affiliations

J C Schymick*, P Momeni, J Elder, A B Singleton, J Hardy, Laboratory of Neurogenetics, National Institute of Aging, NIH, Bethesda, Maryland, USA Y Yang ${ }^{*}$, J Rothstein, Department of Neurology and Neuroscience, Johns Hopkins University, Baltimore, Maryland, USA

P M Andersen*, C Dahlberg, Department of Neurology and Clinical Neuroscience, Umeå University Hospital, Umeå, Sweden

J P Vonsattel, Taub Institute for Research on Alzheimer's Disease and the Aging Brain, and Department of Pathology, College of Physicians and Surgeons, Columbia University, New York, New York, USA

M Greenway, O Hardiman, Department of Neurology, Beaumont Hospital and Royal College of Surgeons in Ireland, Dublin, Ireland

A Chiò, Department of Neuroscience, University of Torino, Torino, Italy

G Restagno, Molecular Genetics Unit, Department of Clinical Pathology,

Children's Hospital, Torino, Italy
} 
W Robberecht, Laboratory of Neurobiology, University of Leuven, Campus Gasthuisberg, Leuven, Belgium

O Mukherjee, A Goate, Washington University Alzheimer's Disease Research Center, Washington University School of Medicine, St Lovis, Missouri, USA

N Graff-Radford, Department of Neurology, Mayo Clinic College of Medicine, Jacksonville, Florida, USA

R J Caselli, Department of Neurology, Mayo Clinic Scottsdale, Arizona, USA

M Hutton, J Gass, A Cannon, R Rademakers, Department of Neuroscience, Mayo Clinic College of Medicine, Jacksonville, Florida, USA B J Traynor, Section on Developmental Genetic Epidemiology, National Institute of Mental Health, Bethesda, Maryland, USA

*J C Schymick, Y Yang and P M Andersen contributed equally to this work Competing interests: None.

Correspondence to: Bryan J Traynor, Porter Neuroscience Building, Room 1A-1014, 35 Convent Drive, Bethesda, MD 20892, USA;

traynorb@mail.nih.gov

Received 21 October 2006

Revised 26 January 2007

Accepted 7 March 2007

Published Online First 19 March 2007

\section{REFERENCES}

1 Snowden JS, Neary D, Mann DM. Frontotemporal dementia. Br J Psychiatry 2002;180:140-3.
2 Ince PG, Lowe J, Shaw PJ. Amyotrophic lateral sclerosis: current issues in classification, pathogenesis and molecular pathology. Neuropathol Appl Neurobiol 1998;24:104-17

3 Hudson AJ. Amyotrophic lateral sclerosis and its association with dementia, parkinsonism and other neurological disorders: a review. Brain $1981 ; 104: 217-47$

4 Lomen-Hoerth C, Murphy J, Langmore S, et al. Are amyotrophic lateral sclerosis patients cognitively normal? Neurology 2003;60:1094-7.

5 Rosso SM, Donker KL, Baks T, et al. Frontotemporal dementia in The Netherlands: patient characteristics and prevalence estimates from a population-based study. Brain 2003;126:2016-22.

6 Mackenzie IR, Feldman HH. Ubiquitin immunohistochemistry suggests classic motor neuron disease, motor neuron disease with dementia, and frontotemporal dementia of the motor neuron disease type represent a clinicopathologic spectrum. J Neuropathol Exp Neurol 2005:64:730-9.

7 Baker M, Mackenzie IR, Pickering-Brown SM, et al. Mutations in progranulin cause tau-negative frontotemporal dementia linked to chromosome 17. Nature 2006:442:916-19.

8 Cruts M, Gijselinck I, van der Z, et al. Null mutations in progranulin cause ubiquitin-positive frontotemporal dementia linked to chromosome 17q21. Nature 2006;442:920-4

9 Brooks BR. El Escorial World Federation of Neurology criteria for the diagnosis of amyotrophic lateral sclerosis. Subcommittee on Motor Neuron Diseases/ Amyotrophic Lateral Sclerosis of the World Federation of Neurology Research Group on Neuromuscular Diseases and the El Escorial "Clinical limits of amyotrophic lateral sclerosis" workshop contributors. J Neurol Sci 1994; 124(Suppl):96-107.

10 Clinical and neuropathological criteria for frontotemporal dementia. The Lund and Manchester Groups. J Neurol Neurosurg Psychiatry 1994:57:416-18.

11 Gass J, Cannon A, Mackenzie IR, et al. Mutations in progranulin are a major cause of ubiquitin-positive frontotemporal lobar degeneration. Hum Mol Genet 2006;15:2988-3001.

12 Mukheriee O, Pastor P, Cairns NJ, et al. HDDD2 is a familial frontotemporal lobar degeneration with ubiquitin-positive, tau-negative inclusions caused by a missense mutation in the signal peptide of progranulin. Ann Neurol 2006;60:314-22.

\section{bmjupdates +}

bmjupdates+ is a unique and free alerting service, designed to keep you up to date with the medical literature that is truly important to your practice.

bmjupdates+ will alert you to important new research and will provide you with the best new evidence concerning important advances in health care, tailored to your medical interests and time demands.

Where does the information come from?

bmjupdates+ applies an expert critical appraisal filter to over 100 top medical journals A panel of over 2000 physicians find the few 'must read' studies for each area of clinical interest

Sign up to receive your tailored email alerts, searching access and more...

www.bmjupdates.com 